\title{
BMJ Open Lower socioeconomic status, adiposity and negative health behaviours in youth: a cross-sectional observational study
}

\author{
Sarah Lord, ${ }^{1}$ Cedric Manlhiot, ${ }^{1}$ Pascal N Tyrrell, ${ }^{1}$ Stafford Dobbin, ${ }^{2}$ Don Gibson, ${ }^{2}$ \\ Nita Chahal, ${ }^{1}$ Karen Stearne, ${ }^{2}$ Amanda Fisher, ${ }^{2}$ Brian W McCrindle ${ }^{1}$
}

To cite: Lord S, Manlhiot C Tyrrell PN, et al. Lower socioeconomic status, adiposity and negative health behaviours in youth: a crosssectional observational study. BMJ Open 2015;5:e008291. doi:10.1136/bmjopen-2015008291

- Prepublication history for this paper is available online. To view these files please visit the journal online (http://dx.doi.org/10.1136/ bmjopen-2015-008291).

The study originated at The Hospital for Sick Children with data provided by Heart Niagara Inc; the protocol was reviewed and approved by the Research Ethics Boards of the Niagara Catholic District School Board and the District School Board of Niagara

Received 24 March 2015 Accepted 22 April 2015

CrossMark

\footnotetext{
${ }^{1}$ Department of Pediatrics, Labatt Family Heart Centre, University of Toronto, The Hospital for Sick Children, Toronto, Canada

${ }^{2}$ Heart Niagara Inc, Niagara Falls, Ontario, Canada
}

Correspondence to Dr Brian W McCrindle; brian.mccrindle@sickkids.ca

\section{ABSTRACT}

Objective: Understanding obesity and its modifiable risk factors in youth is key to addressing the burden of cardiovascular disease later in life. Our aim was to examine the associations among adiposity, negative health behaviours and socioeconomic status in youth from the Niagara Region.

Design, setting and participants: Cross-sectional observational study of 3467 grade 9 students during their mandatory health and physical education class to investigate the association between socioeconomic status (postal code), self-reported health behaviour and adiposity in the Niagara Region, Ontario, Canada.

Results: Median household income was $\$ 63696$ and overall percentage below the after-tax low-income cutoff was $4.2 \%$. Negative health behaviours (especially skipped meals, lower fruit and vegetable consumption, higher screen time) were associated with lower income neighbourhoods, however, the absolute effect was small. Those participants in the lowest income quintile had a significantly greater body mass index z-score than those in the highest $(0.72 \pm 1.19$ vs $0.53 \pm 1.12)$, but the overall trend across quintiles was not statistically significant. A similar trend was noted for waist-to-height ratio. The lowest income neighbourhoods according to after-tax low-income cut-off had small but statistically significant associations with higher adiposity compared with the middle or highest income neighbourhoods.

Conclusions: Obesity prevention efforts should target modifiable behaviours, with particular attention to adolescents from lower income families and neighbourhoods.

\section{INTRODUCTION}

Obesity in youth is increasingly prevalent. Rates of childhood overweight and obesity in Canada have risen from $15 \%$ in 1979 , to $26 \%$ in $2004 .{ }^{1}$ One cardiovascular disease (CVD) prevention initiative established in 1987 by Heart Niagara Inc is the Healthy Heart Schools' Program in the Niagara

\section{Strengths and limitations of this study}

- Results from universal screening better characterise the relationship between socioeconomic status (SES), negative health behaviours and adiposity in adolescents.

- A review of contributing factors of physical activity, screen time, consumption patterns and diet was undertaken.

- The current study is limited by the use of surrogate population-based SES data for the study participants.

- Variability within a postal code could not be explored due to the limitations of available Census data.

Region, Ontario. The programme identifies adolescents with CVD risk factors through universal screening in schools and provides appropriate referrals to care providers. A previous analysis of Heart Niagara Inc data showed increases in the prevalence of overweight/obesity from $27 \%$ to $32 \%$ in grade 9 students between 2002 and 2008. ${ }^{2}$ The same analysis included an extensive survey of health behaviours and demonstrated that several less favourable health behaviours were significantly associated with increased adiposity: less fruit/vegetable intake and physical activity; and more soft drink consumption, restaurant meals, skipped breakfasts and screen time.

Attention is being paid to identifying environmental contributors to adiposity, including socioeconomic status (SES). A recent systematic review by Shrewsbury and Wardle ${ }^{3}$ confirmed that the associations between SES and adiposity are mainly inverse. In addition to family income, parental education and rural/urban status, the literature advocates for examining income disparity as another important marker of SES. ${ }^{4}{ }^{5}$ Statistics 
Canada's low-income cut-off (LICO) reflects the income below which a family would spend at least a $20 \%$ greater portion of their income than average on necessary food, clothing and shelter. ${ }^{6}$ This is used to define families as 'low income' compared with similar sized families in a defined region, and it is analogous to what other countries refer to as the 'poverty line'. We sought to better characterise the association between SES and CVD risk in terms of adiposity and negative health behaviours in youth in the Niagara Region in order to identify targets for CVD prevention efforts.

\section{METHODS}

The Heart Niagara Inc Healthy Heart Schools' Program is a universal screening programme provided as a curriculum enrichment programme. It identifies adolescents who are at increased risk for CVD and refers such individuals to their primary care providers for assessment. The programme targets all adolescents during their mandatory grade 9 health and physical education class in the geographically and administratively defined Niagara Region, Ontario. The detailed methodology of the Healthy Heart Schools' Program has been published. ${ }^{2}$

Before physical assessment, each student was given a questionnaire regarding family history, health and lifestyle behaviours. All students provided informed assent and parental consent.

Body mass index (BMI) and waist measurements were used as measures of adiposity. BMI values were converted to WHO z-scores, and waist and height measurements to waist-to-height ratios (WHtRs). The following modifiable health behaviours were chosen for analysis, given their significant association with higher adiposity in a past published analysis ${ }^{2}$ : physical activity (sessions/ week with $>30$ min of light activity or $>20$ min of moderate/vigorous activity), screen time (hours/day outside of school spent using computers, playing video games or watching television), restaurant meals/week, soft drinks/ day, fruit and vegetable servings/day and skipped breakfast/week.

Data were collected during the 2009-2010 school year. Participants underwent standardised height and weight measurements by Heart Niagara staff. These values were converted to BMI z-scores using the 2006 growth standards provided by the WHO. ${ }^{7}$ Waist circumference measurement was standardised, concordant with methods used in the National Health and Nutrition Examination Survey, ${ }^{8}$ and then converted to WHtRs.

Socioeconomic data were obtained from Census Canada through the Canadian Socio-Economic Information Management System. Data were sorted by Dissemination Area Unique Identifier (the smallest geographical division) and then matched to postal code. Four socioeconomic variables were chosen: median household income, percentage of population below the after-tax LICO, rural/urban status and percentage with a Bachelor's degree or higher. Median household income was categorised in quintiles according to the panCanadian census data. Data were analysed in terms of after-tax LICO, as this is considered to be the measure that better reflects spending ability. ${ }^{9}$ For feasibility of analysis, the after-tax LICO results were grouped into three categories: (1) areas with $0 \%$ of the population living below the LICO (ie, highest income neighbourhoods); (2) areas with more than $0 \%$ but less than the provincial average living below the after-tax LICO (ie, middle-income neighbourhoods) and (3) areas with the same or higher proportion of the population living below the LICO as compared to the province as a whole (ie, lowest income neighbourhoods). Areas were classified as rural or urban according to Statistics Canada's method for assigning postal codes. ${ }^{10}$ The SES data were then matched by postal code to the participants' data as a surrogate for individual participants' socioeconomic data (which were not available).

\section{Data analysis}

Summary statistics are described by the mean with SD, median with IQR or frequencies. Comparative analyses of health behaviour and adiposity by income quintile and LICO groups were performed using multivariable linear regression models. These models were adjusted for within-school clustering through an autoregressive covariance structure. Wald $\chi^{2}$ (5 levels, 4 degrees of freedom) was used to determine the global statistical difference among all household income quintiles. The lowest quintile was used as the reference against which all other categories were assessed. For LICO, the middle category (ie, areas with more than zero but less than the provincial average living below the after-tax LICO) was used as the reference category. All regression models were adjusted for age, sex, area (rural/urban) and number of siblings (as an estimate of family size). Effect of area (rural/urban) was obtained in the regression models for income quintile; this result would not have differed if the model for LICO group had been used. All analyses were performed using SAS software, V.9.3 (SAS Institute, Cary, North Carolina, USA).

\section{RESULTS}

\section{Population}

A total of 4884 students were enrolled in grade 9 during the study period, and 4104/4884 students (84\%) participated. Postal codes and adiposity data were available for $\mathrm{n}=3494$ students $(85 \%)$. All but six postal codes (27 students) were included, based on a normal distribution for median income, resulting in data analysed for 3467 participants. Included participants ranged from 13 to 16 years of age. The average age of participants was 14.5 \pm 0.4 years, and the gender distribution was equal. The participants were from 32 high schools across all 12 municipalities in the Niagara Region, which comprises a total population of just under 500000 . 


\section{Socioeconomic profile}

Median yearly household income in the study population was \$63 696 (IQR: \$48 183-\$74 784). Using the annual income distribution in Canada as the basis, $24 \%$ of our study sample was in the highest annual income quintile $(\geq \$ 76207), \quad 26 \%$ in the second quintile (\$62 677-\$76 207), 19\% in the third quintile (\$51 259$\$ 62676), 16 \%$ in the fourth quintile (\$41 216-\$51 258) and $15 \%$ in the lowest quintile $(<\$ 41216)$. The median percentage of people living below the after-tax LICO was $4.2 \%$ (IQR: $0-8.3 \%$ ). Of the participants, $42 \%$ were from areas with no households living below the after-tax LICO, $40 \%$ were in the middle income areas and $18 \%$ were in areas with a higher percentage of the population living below the after-tax LICO compared with the provincial value of $10.3 \% .{ }^{6}$ Those living in rural areas made up $26 \%$ of participants, while $74 \%$ lived in urban areas. Among all postal codes, $86 \%$ of the population completed high school and 17\% completed a bachelor's degree or higher.

\section{Health behaviours and SES}

Health behaviours were examined by median household income, percentage of people with a bachelor's degree or higher, after-tax LICO and urban/rural status. Since the percentage of people with a bachelor's degree or higher followed the same trend as household income, with an increase from $12 \%$ to $26 \%$ across the lowest to highest income quintiles, we chose median annual household income for the purposes of our analysis.

Average values for the chosen health behaviours by median household income (grouped by quintile and also shown linearly per $\$ 10000$ increase) are shown in table 1 and by LICO (grouped categorically to reflect lower, middle and higher income neighbourhoods as well as linearly by $1 \%$ change in LICO) in table 2 . All variables showed a statistically significant pattern of association between less favourable behaviours and lower income; all except restaurant meals and physical activity showed the same association when grouped by income quintile. Statistically significant poorer behaviours, compared with the lowest income quintile, were observed at the middle income quintile for soft drink consumption, skipped breakfast and screen time (table 1). Fruit/vegetable consumption was statistically different from the lowest income quintile, for the second highest income quintile onward. Statistical differences in restaurant meals per week and physical activity were observed only between the lowest and highest income quintiles.

Participants from the lower income neighbourhoods were found to consume more soft drinks and skip more breakfasts compared with the middle and higher income neighbourhoods, when participant postal codes were grouped by percentage of people living below the LICO (table 2). The lowest income neighbourhoods also had lower physical activity than the middle LICO group. There was a trend towards more screen time in the lowest income neighbourhoods, though it was not statistically significant. There were no differences among the LICO categories for the number of restaurant meals or fruit/vegetable consumption (table 2).

There were no differences between urban and rural areas regarding fruit and vegetable consumption (parameter estimate rural vs urban (SE): +0.08 (0.06) portions/day, $\mathrm{p}=0.19$; rural: $+0.02 \quad(0.03)$ drinks/day, $\mathrm{p}=0.60)$, number of restaurant meals (rural: $+0.04(0.06)$ meals/week, $\mathrm{p}=0.47$ ) or physical activity (rural: +0.03 (0.22) periods/day, $\mathrm{p}=0.88$ ). Participants from rural areas had significantly less screen time (rural: -0.46 (0.17) hours/day, $\mathrm{p}=0.008$ ) and were less likely to skip breakfast (rural: $-0.21(0.08)$ days/week, $\mathrm{p}=0.009$ ).

\section{Adiposity and SES}

Both measures of adiposity (BMI z-score and WHtR) demonstrated a decreasing trend with higher income quintile, though the trend was not statistically significant (table 1). Only the lowest and highest income quintiles were different from one another. Examining the data by after-tax LICO categories demonstrated a statistically significantly higher BMI z-score and WHtR in the highest LICO percentage (lowest SES; table 2). There were no differences in adiposity between urban and rural area participants.

\section{DISCUSSION}

Our findings confirm the previously reported relationships among lower income, adiposity and poorer health behaviours. ${ }^{3}{ }^{11-13}$ We found a trend towards lower BMI and WHtR with increasing household income, with statistically significant differences when comparing the lowest and highest quintiles. Our analysis of after-tax LICO as a marker of neighbourhood affluence demonstrated that participants from the lowest SES neighbourhoods had slightly higher BMI and WHtR.

Overall, the absolute trends were small, but our findings suggest that differences in adiposity and health behaviours for adolescents are seen among different SES groups even within a relatively affluent region, emphasising the significance of relative SES. Our analysis of SES in the Niagara Region found this area to be relatively well-off, with $4.2 \%$ of the participants living below the after-tax LICO, compared with the provincial and national rates of $10.3 \%$ and $10.8 \%$, respectively. ${ }^{6}$ The percentage of the population below the LICO for the Niagara Region as a whole is most similar to the highest income province of Prince Edward Island (PEI), where percentage below the LICO is $5.4 \% .{ }^{14}$ Compared with Ontario, PEI has a higher proportion of overweight youth but a lower proportion of obese youth. ${ }^{14}$ Similar to another study by Joliffe, ${ }^{15}$ this suggests that SES may play a stronger role in the severity of overweight/obesity than in the prevalence alone. This lends evidence to the suggestion that adolescents from relatively lower SES backgrounds are at higher risk for more severe adiposity compared with people from higher SES backgrounds, 

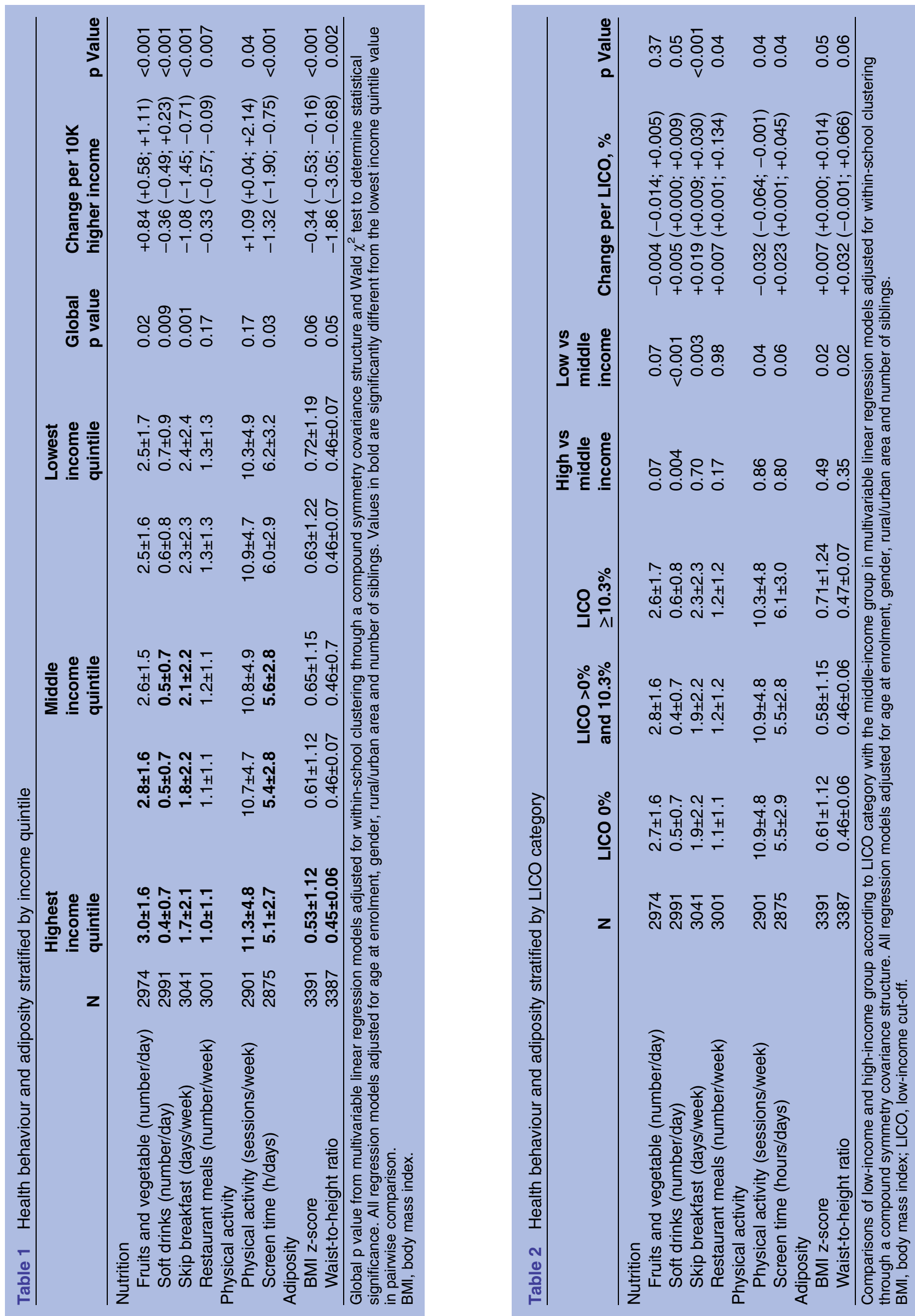

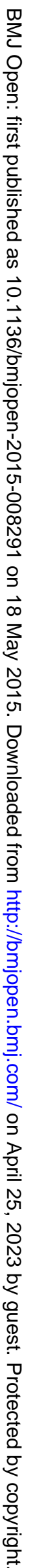


pointing to the need for identifying potential modifiable factors.

Our analysis of SES and health behaviours suggests that the observed associations with adiposity can be at least partially explained by certain health behaviours that followed a similar trend. When examined by LICO, the lower income areas were worse off than the middle and higher income areas in terms soft drink consumption, skipped breakfast and physical activity, which may contribute to the similar pattern found for BMI and WHtR through their common effect on calorie overconsumption or decreased energy expenditure. Increased soft drink consumption and skipping breakfast are both associated with higher daily calorie consumption. ${ }^{11} 12$ There was a trend towards greater screen time in the lowest income neighbourhoods (though it was not statistically significant), and it has been previously shown that increased screen time is associated with increased BMI due to diversion of time away from physical activity, as well as higher calorie intake influenced by advertising of energy-dense foods. ${ }^{13} 1617$ There were no differences among the groups for fruit/vegetable consumption or restaurant meals.

Overall fruit/vegetable consumption was generally low compared with typical healthy eating guidelines, which may explain the former. Whether or not there was a difference in accessibility to affordable restaurants among the different schools is beyond the scope of this analysis, though access to restaurants near schools has been shown to have a significant association with poorer eating habits among adolescents. ${ }^{18}$ Overall, the findings for these health behaviours likely contribute to the similar trend in adiposity through their effects on overconsumption and decreased activity.

There was little difference among behaviours when comparing participants from urban versus rural areas, with slightly more favourable results for screen time and skipped breakfast in rural areas. There was no difference in adiposity between the two groups. Some of the most and least affluent areas of Niagara Region are rural, which likely affected our results. Some previous studies have also suggested that screen time is slightly lower for rural adolescents, with greater availability of space for free play cited as one potential explanation. ${ }^{19}$ However, results for the influence of urban/rural status on adiposity and health behaviours are equivocal, and likely influenced by complexities in SES distribution across various geographical regions.

The current study is limited by the use of surrogate population-based SES data for the study participants; however, there are data that suggest that population and individual SES characteristics are similarly associated with CVD risk. ${ }^{20}$ Variability within a postal code could not be explored due to the limitations of available Census data. The large sample size better lends itself to using indirect SES measures for the purpose of demonstrating a trend, though our findings should be confirmed with more objective measures of health behaviours and individuals' SES.

\section{CONCLUSIONS}

Our findings demonstrate that lower income is related to higher adiposity and more adverse health behaviours for adolescents, even in a relatively affluent population. The observed trend is supported by negative health behaviours as a potential mechanism. Initial targets for CVD prevention in this population should include eating a nutritious breakfast, reducing sugary drink consumption and replacing screen time with physical activity. While most Canadians would benefit from lifestyle changes, those who live in relatively lower income areas should receive particular attention. Positive initiatives such as the Heart Niagara Healthy Heart Schools' Program, which take a population approach to identifying and educating individuals at risk of CVD, reflect the community's acknowledgement of the problem and perhaps serve to reduce adiposity through increased awareness.

Acknowledgements This work was supported by The Canadian Institutes of Health Research and the CIBC Endowed Chair in Child Health Research.

Contributors SL, NC, BWM, KS, SD, DG and AF conceived the study. KS, SD, $\mathrm{DG}$ and AF collected the data. CM, PNT and BWM conducted statistical analysis. SL, CM, PNT, NC and BWM interpreted the data. CM, PNT and SL drafted the manuscript. All authors provided critical revisions and approved the final manuscript.

Funding This research was supported by the Canadian Imperial Bank of Commerce (CIBC) World Markets Children's Miracle Foundation Chair in Child Health Research and the Canadian Institutes of Health Research Team Grant in Childhood Obesity.

\section{Competing interests None declared.}

Ethics approval The study protocol was reviewed and approved by the Research Ethics Boards of the Niagara Catholic District School Board, and the District School Board of Niagara.

Provenance and peer review Not commissioned; externally peer reviewed.

Data sharing statement No additional data are available.

Open Access This is an Open Access article distributed in accordance with the Creative Commons Attribution Non Commercial (CC BY-NC 4.0) license, which permits others to distribute, remix, adapt, build upon this work noncommercially, and license their derivative works on different terms, provided the original work is properly cited and the use is non-commercial. See: http:// creativecommons.org/licenses/by-nc/4.0/

\section{REFERENCES}

1. Shields M. Measured obesity. Overweight Canadian children and adolescents. Statistics Canada Cat. No. 82-620-MWE2005001. 2005.

2. McCrindle BW, Manlhiot C, Millar K, et al. Population trends toward increasing cardiovascular risk factors in Canadian adolescents. J Pediatr 2010;157:837-43.

3. Shrewsbury V, Wardle J. Socioeconomic status and adiposity in childhood: a systematic review of cross-sectional studies 19902005. Obesity 2008;16:275-84.

4. Shearer C, Blanchard C, Kirk S, et al. Physical activity and nutrition among youth in rural, suburban and urban neighbourhood types. Can J Public Health 2012;103:eS55-60.

5. Rossen LM. Neighbourhood economic deprivation explains racial/ ethnic disparities in overweight and obesity among children and adolescents in the USA. J Epidemiol Community Health 2014;68:123-9.

6. Statistics Canada. Low income cut-offs for 2008 and low income measures for 2007. Statistics Canada, 2009. http://www.statcan.gc. $\mathrm{ca} / \mathrm{pub} / 75 \mathrm{f0002m} / 2009002 / \mathrm{s} 2-e n g . h t m$ (accessed 1 May 2012). 
7. de Onis M, Onyango AW, Borghi E, et al. Development of a WHO growth reference for school-aged children and adolescents. Bull World Health Organ 2007;85:660-7.

8. Fryar CD, Ogden CL. Prevalence of underweight among children and adolescents: United States, 2003-2006. National Center for Health Statistics, 2009. http://www.cdc.gov/nchs/data/hestat/ underweight/underweight_children.htm (accessed 30 May 2011).

9. Bloch G, Etches V, Gardner C, et al. Identifying poverty in your practice and community. Ont Med Rev 2008;75:39-73.

10. Statistics Canada. Postal code conversion file: reference guide. Statistics Canada, 2011. http://www.statcan.gc.ca/pub/92-153-g/ 2011002/tech-eng.htm (accessed 1 Jun 2012).

11. Timlin MT, Pereira MA, Story M, et al. Breakfast eating and weight change in a 5-year prospective analysis of adolescents: project EAT (eating among teens). Pediatrics 2008;121:e638-45.

12. Ludwig DS, Peterson KE, Gortmaker SL. Relation between consumption of sugar-sweetened drinks and childhood obesity: a prospective, observational analysis. Lancet 2001;357: 505-8.

13. Dietz WH Jr, Gortmaker SL. Do we fatten our children at the television set? Obesity and television viewing in children and adolescents. Pediatrics 1985;75:807-12.
14. Poverty Backgrounder. Research and statistics about poverty in Nova Scotia. Government of Nova Scotia. 2008. http://www.gov.ns. $\mathrm{ca} / \mathrm{coms} /$ department/backgrounders/poverty/Poverty_Stats-May2008. pdf (accessed 31 May 2012)

15. Jolliffe D. Overweight and poor? On the relationship between income and the body mass index. Econ Hum Biol 2011;9:342-55.

16. Crespo CJ, Smit E, Troiano RP, et al. Television watching, energy intake, and obesity in US children: results from the third National Health and Nutrition Examination Survey, 1988-1994. Arch Pediatr Adolesc Med 2001:155:360-5.

17. Van den Bulck J, Van Mierlo J. Energy intake associated with television viewing in adolescents, a cross sectional study. Appetite 2004:43:181-4.

18. Davis B, Carpenter C. Proximity of fast-food restaurants to schools and adolescent obesity. Am J Public Health 2009;99:505-10.

19. Dollman J, Maher C, Olds TS, et al. Physical activity and screen time behaviour in metropolitan, regional and rural adolescents: a cross-sectional study of Australians aged 9-16 years. J Sci Med Sport 2012;15:32-7.

20. Tremblay MS, Willms JD. Is the Canadian childhood obesity epidemic related to physical inactivity? Int J Obes Relat Metab Disord 2003;27:1100-5. 